\title{
Protective Mechanism of Adipose-Derived Stem Cells in Remodelling of the Skin Stem Cell Niche During Photoaging
}

\author{
Meihua Gong Pan Zhang Chunyang Li Xu Ma Daping Yang \\ Division of Plastic Surgery, the 2nd Hospital of Harbin Medical University, Harbin, China
}

\section{Key Words}

Photoaging $\cdot$ Stem cell niche $\cdot$ Extracellular matrix $\bullet$ BMP signalling pathway $•$ Adipose-derived stem cells $•$ Bone morphogenetic protein $4 \cdot$ Paracrine pathway

\begin{abstract}
Background/Aims: Skin photoaging is primarily caused by the functional attrition of skin stem cells. The skin stem cell niche plays an important role in maintaining stem cell survival and behaviour. In our study, we hypothesized that UVB irradiation induces skin photoaging by changing skin stem cell niches and that transferred adipose-derived stem cells (ADSCs) can remodel the niches by affecting the BMP signalling pathway and transdifferentiating into skin stem cells. Methods: Sixty-four C57BL/6J mice were divided into the following groups: a control group, the UVB group and the UVB+ADSCs group. Western blot assays, immunofluorescence analysis and real-time PCR were used to measure differences in the expression of niche components among the three groups. Furthermore, we tested whether transplanted ADSCS express skin stem cell markers, such as p63, $\alpha 6$-integrin and CD34. Results: The expression levels of Bmp4, its downstream factors Smad1 and MAPK1 and a regulatory factor of the niche, i.e., NFATc1, were lower in the UVB group than were those in the control group $(P<0.05)$ but higher in the UVB+ADSCs group than were those in the UVB group $(P<0.05)$. Compared with Bmp4, Nanog (a downstream factor of Bmp4), and MMP13 (a regulatory factor of the niche), ICAM-1 (a proinflammatory gene), p63 (a basal transcription factor), $\beta 1$-integrin, Mtnr1a and Tyr (melanogenesis-related factors) showed the opposite expression trends $(P<0.05)$. Bmp2 and Collagen IV levels did not significantly change among the three groups $(P>0.05)$. Skin stem cell markers, such as p63, $\alpha 6$-integrin and CD34, were coexpressed in the ADSCs, which suggested the ADSCs may transdifferentiate into skin stem cells. Conclusion: We found that UVB irradiation results in typical photoaging signs by altering skin stem cell niches and that Bmp4 was a key factor in BMP signalling in hair follicles. ADSCs reversed these typical photoaging signs by remodelling skin stem cell niches through BMP4 pathway modulation and transdifferentiation into skin stem cells.




\section{Introduction}

As the largest organ in the body, the skin provides a protective barrier against biological, physical and chemical damage. Impaired tissues and ageing cells are restored by continuous self-renewal of the skin. Tissue homeostasis maintenance depends on stem cell activity and diminishes over time or through constant UVB exposure. Reduced trauma repair ability is a typical hallmark of skin ageing. Other features, such as wrinkles, pigmentation and immunity, are also affected by ageing. Although there is no consensus on the exact mechanism of ageing, it is generally held that functional attrition of skin stem cells is responsible [1]. Whether stem cells or the local environment is responsible for skin cell behaviour remains unclear. Skin stem cells are present in the epidermis, sebaceous glands and adult hair follicles [2]. As a mini-organ, the hair follicle is an ideal model to explore the nature of stem cells due to its lifelong cyclic regeneration [3]. In this study, the hair follicle was used as a research model to investigate local environmental changes in skin stem cells during photoaging.

Stem cells reside in a niche, a dynamic microenvironment that is essential for stem cells to maintain periodic regeneration and differentiation. Stem cell niches are predominantly composed of an extracellular matrix (ECM) and various regulatory factors (Fig. 1). The ECM is an important component of the niche and interacts with stem cells through a complex network of macromolecules that regulates stem cell proliferation and differentiation [4]. The ECM maintains stem cells in appropriate locations in niches and provides the stiffness required for stem cell survival and behaviour [5]. Alteration of ECM structure and composition, especially that of collagen fibres, during ageing will reduce tissue stiffness and elasticity. In addition to the microenvironment, stem cells are also regulated by macroenvironmental signals to adapt to physiological changes [3]. Compared with other signalling pathways, the Wnt (activator)/BMP (inhibitor) signalling pathway has been shown to have a major role in modulating periodic hair follicle regeneration [6]. Plikus et al. found that the periodic expression of extrafollicular Bmp2 and Bmp4 was regulated by the surrounding microenvironment, or niche [7]. The regeneration cycle of stem cells can be delayed due to changes in signalling factors in ageing mice [3]. Ageing weakens stem cell capacity by altering the microenvironmental structure and macroenvironmental signalling pathways in niches.

Skin ageing occursthrough two processes, intrinsic ageing and extrinsicageing. Ultraviolet $B$ (UVB) radiation exposure, the major cause of extrinsic ageing, is known as photoaging. UVB irradiation has a significant impact on collagen production and pigmentation and is responsible for skin thickening, mottled pigmentation, wrinkling and decreased elasticity $[8,9]$. Various surgical and nonsurgical methods have been used to treat skin photoaging. Compared with other methods, the transplantation of adipose-derived stem cells (ADSCs) is more successful in improving skin photoaging. However, the antiphotoaging mechanism of ADSCs is unclear. In this study, we aimed to explore the mechanism by which ADSCs remodel the skin stem cell niche during photoaging.

\section{Materials and Methods}

\section{Animal studies}

Animal experimental protocols were approved by the Institutional Animal Care and Use Committee of the 2nd Hospital of Harbin Medical University. All experiments were performed according to the Guidelines for Animal Experimentation of the 2nd Hospital of Harbin Medical University. All efforts were made to minimize animal suffering. C57BL/6J mice were obtained from the Animal Experiment Centre of the 2nd Hospital of Harbin Medical University. All mice were maintained in an air-conditioned room at $24 \pm 2{ }^{\circ} \mathrm{C}$ and $50 \%$ humidity, with a $12 \mathrm{~h} / 12 \mathrm{~h}$ light/dark cycle (lights on at 7:00 a.m.). All mice were allowed free access to water and food. 


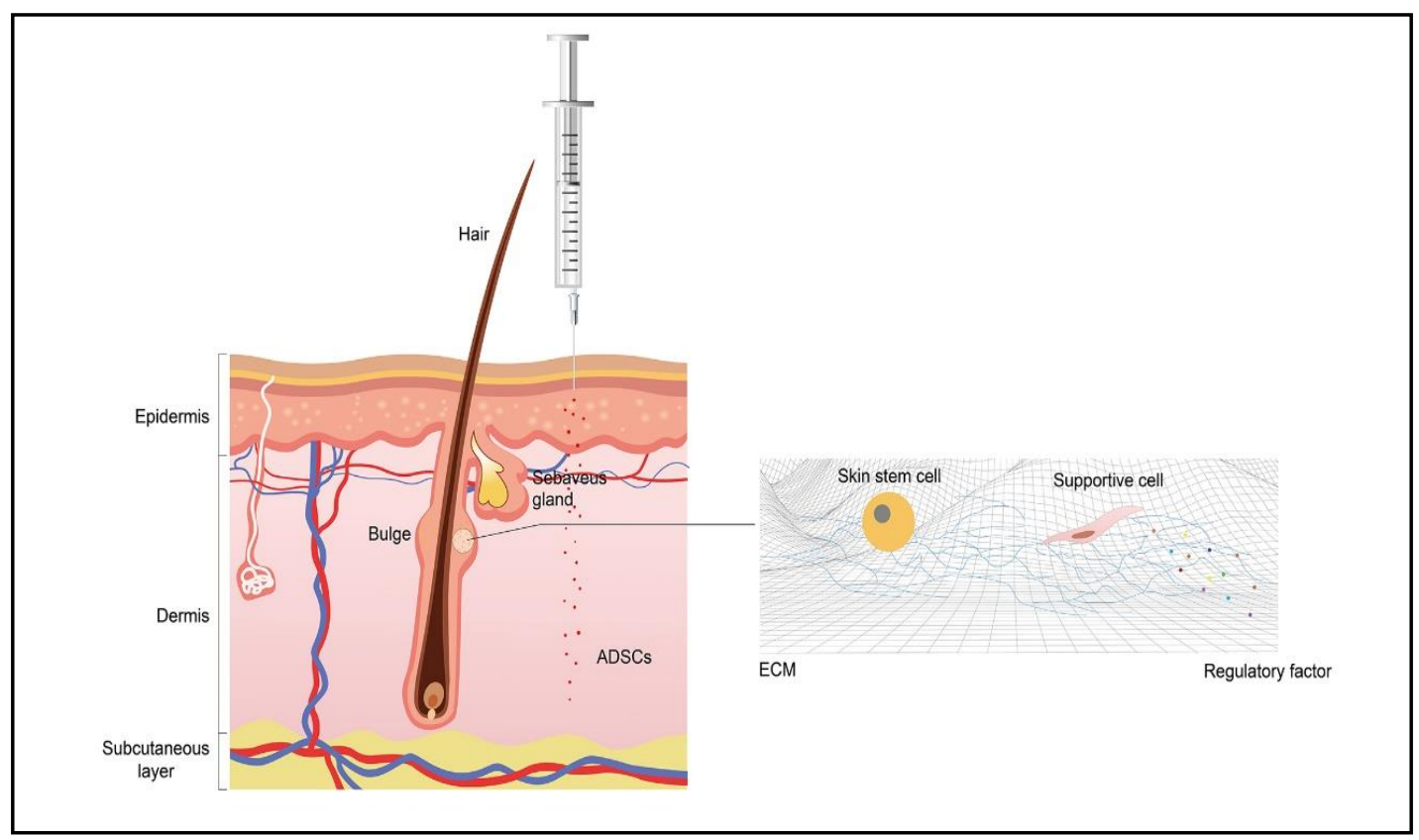

Fig. 1. Diagram of the hair follicle. (Left) Skin is composed of three layers, the epidermis, dermis and subcutaneous layer. Hair follicles are located in the dermis. Hair follicle stem cells (HFSCs) are present in the bulge, surrounding the hair shaft just below the sebaceous gland. After CM-Dil staining, ADSCs were injected into the intradermal and subdermal layers of mouse dorsal skin. (Right) Local microenvironment of HFSCs. Stem cells reside in a dynamic microenvironment called a niche. Niches are primarily composed of ECM and various regulatory factors.

\section{Establishment of the photoaging model and grouping}

Six-week-old female C57BL/6J mice were used to establish photoaging models. Mouse back hairs were removed with an electric shaver once a week, and the backs of the mice were exposed to a narrow-band UVB lamp (wavelengths of 290-320 nm, peak wavelength was $312 \mathrm{~nm}$, TL20 W/12; Philips, Eindhoven, The Netherlands). During exposure, the mice could move freely within the closed box. The distance from the lamp to the bottom of the box was $25 \mathrm{~cm}$. Two irradiation methods, chronic irradiation and rapid irradiation, were selected. Two groups, each including 5 mice, were formed. The chronic irradiation group was exposed to UVB 5 times weekly for 8 weeks (Fig. 2), resulting in a characteristic photoaged morphometric result. The irradiation intensity, represented by the minimal erythemal dose (MED), was set at 1 MED during the first 2 weeks $\left(60 \mathrm{~mJ} / \mathrm{cm}^{2}, 18 \mathrm{~min}\right)$ and was elevated to $2 \mathrm{MED}\left(120 \mathrm{~mJ} / \mathrm{cm}^{2}, 36 \mathrm{~min}\right)$ in the third week, 3 $\operatorname{MED}\left(180 \mathrm{~mJ} / \mathrm{cm}^{2}, 54 \mathrm{~min}\right)$ in the fourth week and $4 \operatorname{MED}\left(240 \mathrm{~mJ} / \mathrm{cm}^{2}, 72 \mathrm{~min}\right)$ during the fifth to eighth weeks of the experiment $[10,11]$. The total UVB dose was approximately 115 MED $\left(6.9 \mathrm{~J} / \mathrm{cm}^{2}\right)$. The rapid irradiation group was exposed to UVB 72 min daily for 4 weeks, and the total UVB dose was the same as that in the chronic irradiation group. Following irradiation, haematoxylin and eosin (HE) staining showed that both models had obvious signs of photoaging (Fig. 3A-C). After an interval of 45 days without any treatment, we unexpectedly observed local hair-loss zones in the area of irradiated skin of the mice in the chronic irradiation group (Fig. 3D-E). The photoaging models used in the subsequent experiments were all established by chronic irradiation. Sixty-four C57BL/6J mice were divided into the following groups: 1) control group - normal mice injected with $0.5 \mathrm{ml}$ phosphate-buffered saline (PBS); 2) UVB group - mice exposed to UVB irradiation and injected with $0.5 \mathrm{ml} \mathrm{PBS}$; and 3) UVB+ADSCs group - mice exposed to UVB irradiation and injected with $5 \times 10^{6}$ ADSCs suspended in $0.5 \mathrm{ml}$ PBS.

\section{ADSCs isolation and culture}

Adipose tissues were obtained from the inguinal tract of 7-day-old C57BL/6J mice. The tissues were washed and digested with $0.1 \%$ type I collagenase (Worthington, Lakewood, NJ, USA) for 45 min at $37^{\circ} \mathrm{C}$ with constant shaking. Then, the tissues were centrifuged for $10 \mathrm{~min}$ at $1200 \mathrm{rpm}$, and the mature adipocytes were discarded. ADSCs were obtained and cultured in dishes with Dulbecco's modified Eagle's 


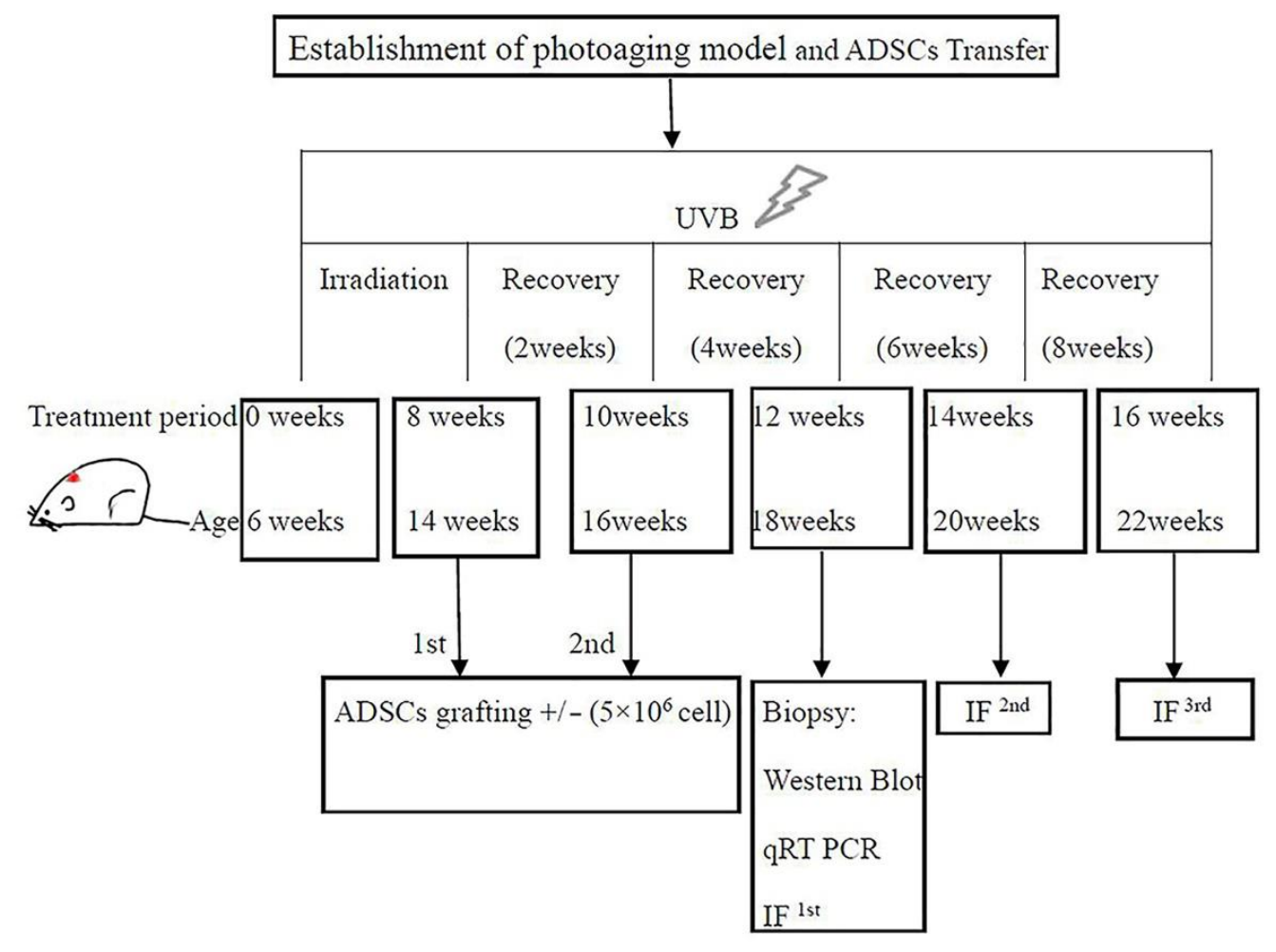

Fig. 2. Establishment of photoaging models and ADSCs transplantation. Six-week-old female C57BL/6J mice were used to establish photoaging models. After 8 weeks of chronic irradiation, the photoaging models were established. Then, $5 \times 10^{6}$ ADSCs were injected into the intradermal and subdermal layers of the mouse dorsal skin at the irradiated area $(1 \mathrm{~cm} \times 1 \mathrm{~cm})$ with a 30 -gauge needle twice at a 14-day interval. Skin sample sections were obtained from the three groups at 2, 4, and 6 weeks after injection.

medium containing 10\% foetal bovine serum (Invitrogen-Gibco, Grand Island, NY, USA) and 1\% antibiotics/ antimycotics (Welgene Inc., Daegu, Korea) at $37^{\circ} \mathrm{C}$ in a $5 \% \mathrm{CO}_{2}$ incubator.

\section{Identification of ADSCs immunophenotype via flow cytometry}

Cultured ADSCs at passage 3 were analysed via fluorescence-activated cell sorting (FACS) to determine the expression of cluster of differentiation CD31, CD34, CD45, CD73, CD90 and CD105. The cells were resuspended in PBS and then incubated with anti-mouse fluorescein isothiocyanate (FITC)-labelled monoclonal antibodies against CD31 (1:200; Miltenyi Biotec; 130-102-519), CD34 (1:200; Miltenyi Biotec; 130-117-775) and CD45 (1:200; Miltenyi Biotec; 130-110-658) and anti-mouse phycoerythrin (PE)labelled monoclonal antibodies against CD73 (1:200; Miltenyi Biotec; 130-102-616), CD90 (1:200; Miltenyi Biotec; 130-102-489) and CD105 (1:200; Miltenyi Biotec; 130-102-548) for $30 \mathrm{~min}$ at $4^{\circ} \mathrm{C}$. FITC-labelled immunoglobulin (Ig)G-stained cells were used as negative controls. Cells were analysed using a FACScan flow cytometer (BD Facs Vantage SE; BD Biosciences, USA). Data were processed with CellQuest Pro software (BD Biosciences).

\section{Multilineage differentiation of ADSCs}

The ability of ADSCs to differentiate into adipocytes and osteoblasts was assessed with an Adipogenesis Differentiation Kit (MesenCult ${ }^{\mathrm{TM}}$ Adipogenic Stimulatory Supplement, 05503, Stem Cell Technologies, Canada) and an Osteogenesis Differentiation Kit (MesenCult ${ }^{\mathrm{TM}}$ Stimulatory kit, 05504, Stem Cell Technologies, Canada) according to the manufacturer's instructions. After 3 weeks of differentiation, Oil Red $\mathrm{O}$ and Alizarin Red S were used to assess adipogenic differentiation and osteogenic differentiation, respectively.

\section{KARGER}




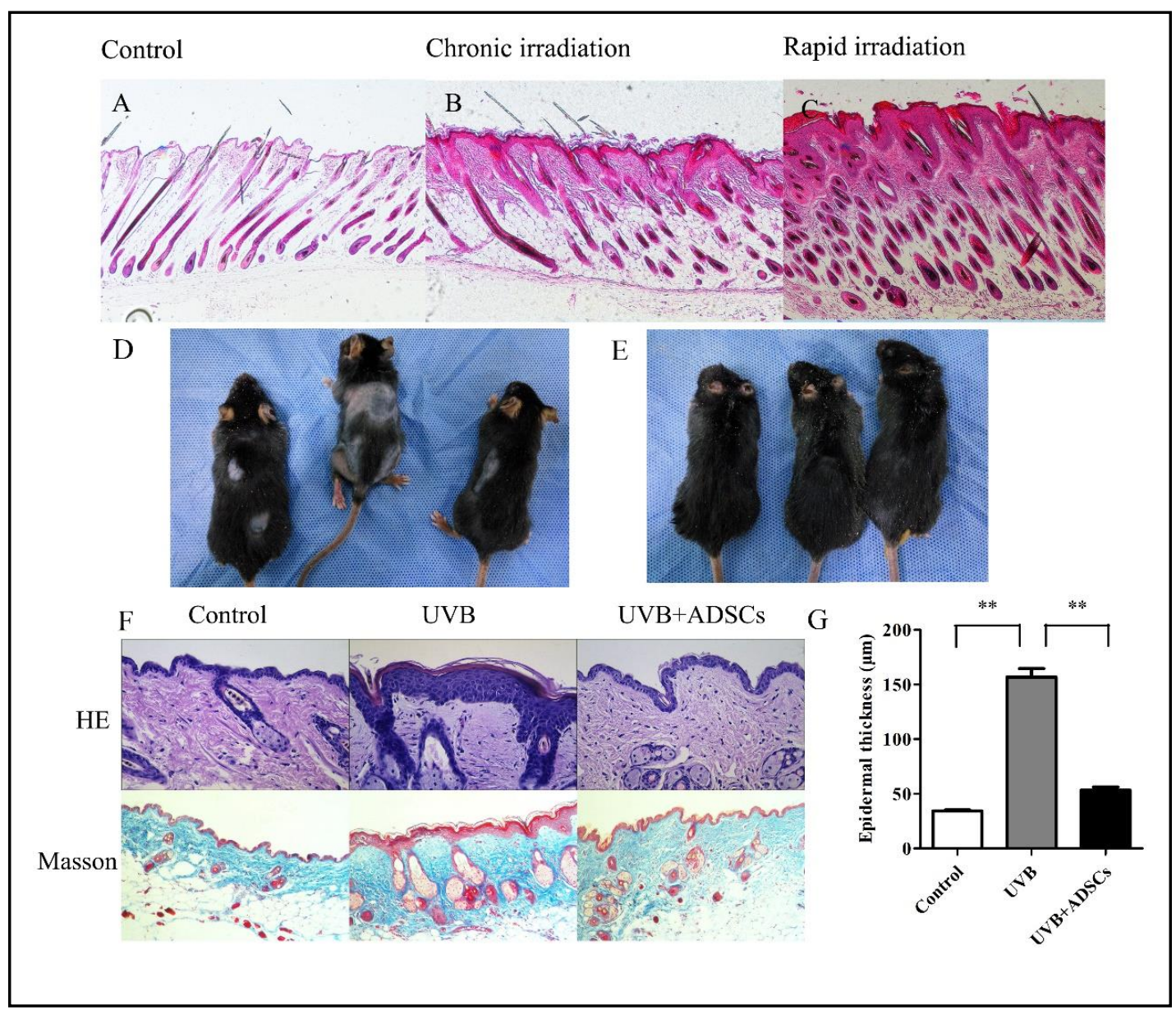

Fig. 3. (A-C) Comparison of rapid and chronic irradiation. After 4 weeks of rapid irradiation and 8 weeks of chronic irradiation, the results of HE staining showed that the mice in the UVB and UVB+ADSCs groups displayed obvious epidermal thickening. (D) After an interval of 45 days without any treatment, local hairloss zones were observed in the UVB-irradiated area in the mice in the chronic irradiation group. (E) There were no local hair-loss zones in the UVB-irradiated area in the mice in the rapid irradiation group (F-G). Histological changes among the three groups. UVB irradiation induced epidermal layer incrassation and hyperkeratosis, and in the UVB group, the cell level was unclear and the dermis layer fibres were not well proportioned. HE staining revealed that the epidermal thickness was significantly increased in the UVB group, while the ADSCs transplantation significantly reduced the epidermal thickness, which approached the normal level observed in the UVB+ADSCs group $\left({ }^{*} \mathrm{P}<0.05,{ }^{* *} \mathrm{P}<0.01\right)$.

Table 1. Sequences of the primers and probes used for RT-PCR

\begin{tabular}{lcc}
\hline Gene & Forward primer $\left(5^{\prime}->3^{\prime}\right)$ & Reverse primer $\left(5^{\prime}->3^{\prime}\right)$ \\
\hline Bmp2 & CAGTCATTCCACCCCACATCA & AAACGAGAAAAGCGTCAGCC \\
Smad1 & CGGTTCTTATTGTTGGACGGA & CAGCAGAGGGATGTTCAGGC \\
MAPK1 & GAGCCTGTTCAACTTCAATCCTC & TTTATGGTCCAGGCGATGAAG \\
MMP-13 & AAGGTCACGGGATGGATGTTC & TTTATGGTCCAGGCGATGAAG \\
Nanog & GCAGAAAGTCCTCCCCGAAGT & GCATCCATTGCAGCTATCCCC \\
NFATC-1 & TGACTTGGACGGGGCTGGTTA & ATCGGCGGGAAGAGATGGTG \\
ICAM-1 & GGAAGGGAGCCAAGTAACTGTGAAG & GAGCGGCAGAGCAAAAGAAGC \\
Mtnr1a & CTGACAGACTGGGTGAAGGTA & CAGCCTCAAGTACGACAAAAT \\
Tyr & CTCAGCCCAGCATCCTTCTTCTC & CGTAATAGTGGTCCCTCAGGTGTTC \\
GAPDH & AAGAAGGTGGTGAAGCAGG & GAAGGTGGAAGAGTGGGAGT \\
\hline
\end{tabular}




\section{Cellular Physiology Cell Physiol Biochem 2018;51:2456-2471 and Biochemistry Published \begin{tabular}{l|l} 
DOI: 10.1159/000495902 & $\begin{array}{l}\text { ( ) } 2018 \text { The Author(s). Published by S. Karger AG, Basel } \\
\text { www.karger.com/cpb }\end{array}$
\end{tabular}}

Gong et al.: Remodelling of the Skin Stem Cell Niche During Photoaging

\section{MTT proliferation assay}

An MTT assay was performed to examine cell proliferation. Chloromethyl-dialkylcarbocyanine (CMDil)-labelled and unlabelled ADSCs were seeded separately in a 96-well plate at a density of $1 \times 10^{3}$ cells/ well in triplicate for eight days and incubated at $37^{\circ} \mathrm{C}$ with $5 \% \mathrm{CO}_{2}$. Every half day, $20 \mu \mathrm{l}$ of MTT $(5 \mathrm{mg} / \mathrm{ml}$ PBS; pH 7.4; C0009, Beyotime, China) was added to the cells for $4 \mathrm{~h}$. The supernatants were then discarded, and $100 \mu \mathrm{l}$ dimethyl sulfoxide (Sigma-Aldrich, Saint Louis, Missouri, USA) was added to each well for $10 \mathrm{~min}$. The optical density (OD) of the samples was measured at an absorbance wavelength of $490 \mathrm{~nm}$ (Microplate Spectrophotometer, TECAN, REF: 30050303, SN: 1406005368). The assay was repeated three times.

\section{Subcutaneous injection of ADSCs}

Cultured ADSCs were stained with CellTracker ${ }^{\mathrm{TM}}$ CM-Dil (C7000, Invitrogen, USA) according to the manufacturer's instructions. Briefly, diluted CM-Dil solution was added directly into PBS to generate the working solution. ADSCs were incubated in the working solution for $5 \mathrm{~min}$ at $37^{\circ} \mathrm{C}$ and then for an additional $15 \mathrm{~min}$ at $4^{\circ} \mathrm{C}$. After labelling, ADSCs were injected into the dorsal skin of mice in the UVB+ADSCs group. A total of $5 \times 10^{6}$ ADSCs suspended in $0.5 \mathrm{ml}$ PBS were injected into the intradermal and subdermal layers of the mouse dorsal skin in the irradiated area $(1 \mathrm{~cm} \times 1 \mathrm{~cm})$ with a 30 -gauge needle. The control and UVB groups were injected with an equal volume of PBS using the same method. The second injection was carried out at 14-day intervals (Fig. 2).

\section{Skin samples}

All mice were sacrificed by inhalational anaesthetic overdose. Skin samples $(1 \mathrm{~cm} \times 1 \mathrm{~cm})$ were obtained from the injection sites of the three groups. After ADSCs transplantation, 1) skin samples harvested at 2 weeks from each group were placed in a liquid nitrogen tank for subsequent extraction of RNA and protein $(\mathrm{n}=6) ; 2)$ skin samples harvested at 2 weeks from each group were placed in $4 \%$ formaldehyde solution $(\mathrm{pH}=7.4)$ for subsequent $\mathrm{HE}$ and Masson's trichrome staining ( $\mathrm{n}=6)$; and 3) skin samples harvested from each group were treated with gradient sucrose dehydration for subsequent immunofluorescence analysis ( $\mathrm{n}=2$, at 2, 4, and 6 weeks) (Fig. 2).

\section{Histological analysis}

Skin epidermal thickness and collagen fibres were assessed via histology. After being fixed and embedded in paraffin, skin sections from the three groups underwent Hematoxylin \& Eosin staining (HE) and Masson's trichrome staining. On HE-stained slices, the thickness of the epidermis was measured at five places in one slice, and the mean values were compared. The dermal collagen density was identified with Masson's trichrome staining.

\section{Immunofluorescence analysis}

Changes in the target proteins, including p63, $\alpha 6$-integrin, Bmp2, Bmp4, Collagen IV and CD34, were identified by immunofluorescence analysis. Skin sample sections were dehydrated with $20 \%$ sucrose for $6 \mathrm{~h}$ and then dehydrated with $30 \%$ sucrose overnight. Dehydrated samples were embedded in optimal cutting temperature compound (Sakura Finetechnical Co., Ltd., Tokyo, Japan). The embedded samples were snapfrozen in a $-80^{\circ} \mathrm{C}$ fridge. Frozen skin samples were cut into 6-mm-thick sections. The frozen slices were soaked in PBS for $5 \mathrm{~min}$, treated with Triton X-100 for 10 min and blocked with 3\% BSA for $0.5 \mathrm{~h}$, followed by incubation at $4^{\circ} \mathrm{C}$ overnight with appropriate dilutions of the following primary rabbit monoclonal antibodies: anti-p63 (1:200; ab53039; Abcam), anti- $\alpha 6$-integrin (1:100; ab18151; Abcam), anti-BMP2 (1:50; ab14933; Abcam), anti-BMP4 (1:50; ab39973; Abcam), anti-Collagen IV (1:50; ab6586; Abcam), and anti-CD34 (1:50; ab81289; Abcam). After three washes with PBS, the skin samples were incubated with the secondary antibody DyLight 488 AffiniPure goat anti-Rabbit IgG $(\mathrm{H}+\mathrm{L})(\mathrm{A} 23220$; Abbkine; China) for $1 \mathrm{~h}$ at $37^{\circ} \mathrm{C}$. Cell nuclei were stained with $4^{\prime}, 6$-diamidine- $2^{\prime}$-phenylindole dihydrochloride (DAPI) (C1002, Beyotime, China) and blocked with glycerol. A confocal microscope (FluoView FV100, Olympus, Tokyo, Japan) was used to analyse the results. 


\section{Cellular Physiology Cell Physiol Biochem 2018;51:2456-2471 \begin{tabular}{l|l|l} 
and Biochemistry & Dublished online: 8 December 2018 & $\begin{array}{l}\text { (c) } 2018 \text { The Author(s). Published by S. Karger AG, Basel } \\
\text { www.karger.com/cpb }\end{array}$
\end{tabular}}

Western blot assays

The protein levels of p63, $\beta 1$-integrin, Bmp2, Bmp4 and Collagen IV in the skin tissues were analysed by Western blotting. Skin samples from the three groups were lysed using RIPA buffer and centrifuged for $10 \mathrm{~min}$ at $15000 \mathrm{~g}$ and $4^{\circ} \mathrm{C}$ to extract total protein. Then, $20 \mu \mathrm{g}$ of total protein were resolved via SDS-PAGE and transferred to a PVDF membrane, followed by incubation overnight at $4{ }^{\circ} \mathrm{C}$ with $4 \mathrm{ml}$ of the appropriate dilutions of the following primary rabbit monoclonal antibodies: anti-p63 (1:2000; ab53039; Abcam), anti-31-integrin (1:5000; ab179471; Abcam), anti-BMP2 (1:1000; ab14933; Abcam), anti-BMP4 (1:2000; ab39973; Abcam), anti-Collagen IV (1:1000; ab6586; Abcam), and anti- $\beta$-tubulin (1:10000; YM3030, Immunoway) as an internal control. Then, the membranes were washed three times and incubated at room temperature for $2 \mathrm{~h}$ with HRP-conjugated goat anti-rabbit IgG $(\mathrm{H}+\mathrm{L})$ at a dilution of 1:10000 (S004, TDY, China). After the membranes were washed three times, the blots were visualized with ECL.

\section{Real-time polymerase chain reaction (RT-PCR)}

Total RNA was extracted using TRIzol (Roche, Switzerland) and quantified spectrophotometrically. Quantification of the Bmp2, Smad1, Mapk1, MMP13, Nanog, NFATC1, ICAM-1, Mtnr1a, and Tyr gene expression levels was performed via RT-PCR, and the results are reported relative to the GAPDH expression level (Table 1). Then, $5 \mu \mathrm{g}$ of total RNA was subjected to first-strand cDNA synthesis using a PrimeScript RT reagent kit (Roche, Switzerland) at $55^{\circ} \mathrm{C}$ for $30 \mathrm{~min}$ and $85^{\circ} \mathrm{C}$ for $5 \mathrm{~min}$. Real-time PCR amplifications were performed on an ABI PRISM 7500 Sequence Detection System with StepOnePlus ${ }^{\mathrm{TM}}$ (ABI, USA). The PCR program was set as follows: $95^{\circ} \mathrm{C}$ for $10 \mathrm{~min}$, followed by 40 cycles of $95^{\circ} \mathrm{C}$ for $15 \mathrm{~s}$ and $60^{\circ} \mathrm{C}$ for $60 \mathrm{~s}$. The relative expression levels of target genes were calculated using the $2^{-\Delta \Delta \mathrm{Ct}}$ method and determined as the fold increase over the expression in controls. All the reactions were repeated at least three times.

\section{Statistical analysis}

All experiments were performed independently at least three times. The results were analysed initially using one-way analysis of variance (ANOVA). When the ANOVA indicated differences among the groups, pairwise comparisons of the control group and the UVB+ADSCs group versus the UVB group were performed using the Dunnett q' statistic. Summary statistics are presented as the mean \pm SD. Differences were considered statistically significant for $P<0.05$.

\section{Results}

Chronic irradiation was superior to rapid irradiation in the establishment of a photoaging model

Typical photoaging characteristics appeared in the mice in the chronic irradiation group and the rapid irradiation group (Fig. 3A-C). While the mice in the chronic irradiation group showed local hair-loss zones in the irradiated region, the hair growth in the rapid irradiation group was normal (Fig. 3 D-E). Compared with the rapid irradiation method, the chronic irradiation method was found to be more suitable for the establishment of a photoaging model.

UVB-induced histological changes were improved by ADSCs transplantation

Epidermal thickness and collagen fibre content in the skin were assessed by HE and Masson's trichrome staining. UVB irradiation induced epidermal layer incrassation, hyperkeratosis, an ill-defined cell layer, and poor proportioned dermis layer fibres (Fig. $3 F$ ). In contrast, with the UVB group, the UVB+ADSCs group showed significantly reduced epidermal thickness, which approached the normal level $(P<0.05)$ (Fig. 3F-G). Collagen fibres, which were stained blue, were identified by Masson's trichrome stain (Fig. 3F). These results suggest that ADSCs can improve epidermal thickening caused by UVB irradiation. 







\section{Cellular Physiology

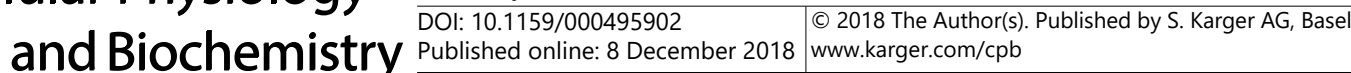

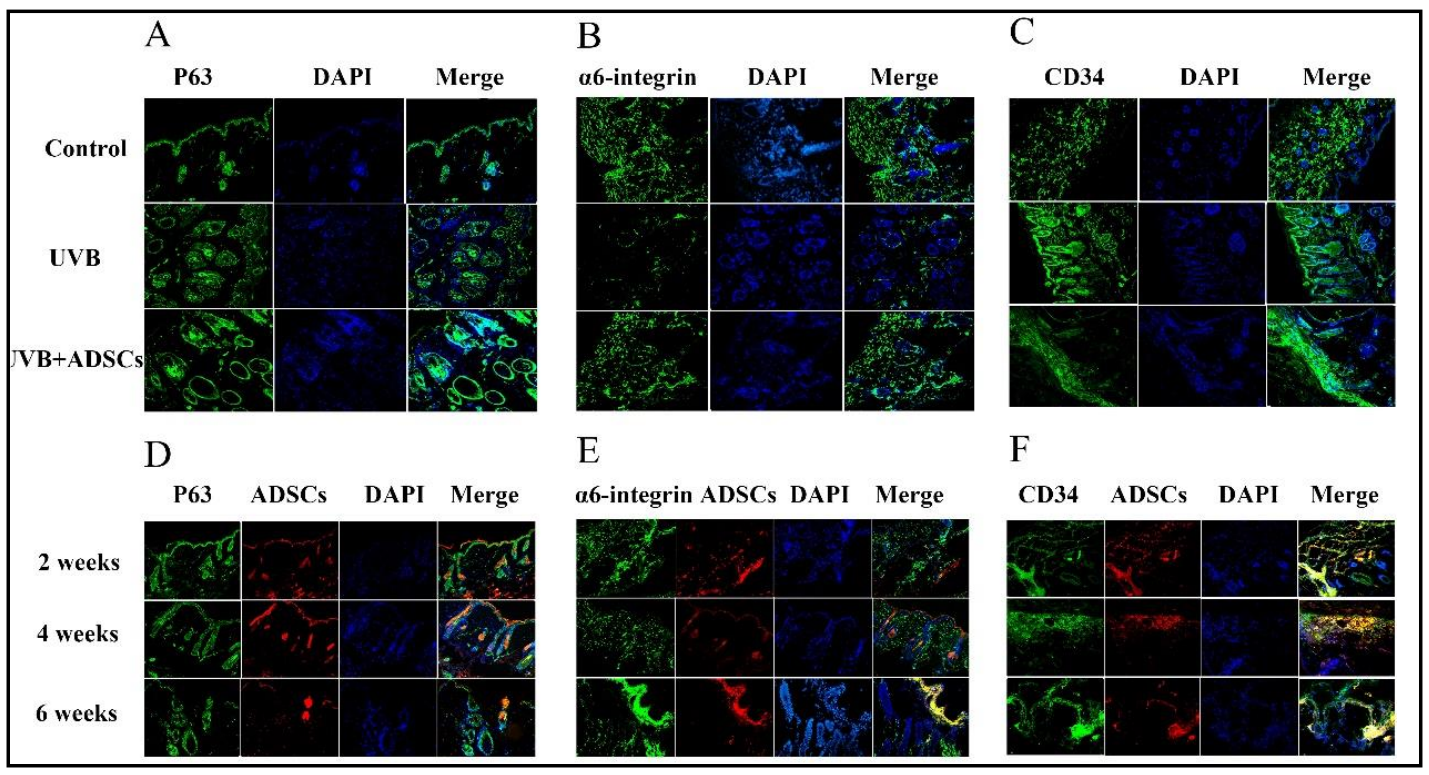

Fig. 5. Coexpression of epithelial cell markers in ADSCs. Fluorescence images of staining (green) for the epithelial cell markers p63, $\alpha 6$-integrin and CD34. Red and blue fluorescence regions represent ADSCs and nuclei, respectively. Merged fluorescence images of skin tissues demonstrate that the epithelial cell markers are coexpressed in ADSCs. Six weeks later, ADSCs stained with red fluorescence were still observed in the skin.

\section{ADSCs characteristics}

ADSCs were characterized by profiling surface CD marker expression. Flow cytometry analysis showed that ADSCs exhibited positive CD73, CD90 and CD105 expression and negative CD31, CD34 and CD45 expression (Fig. 4A).

MTT assays demonstrated that the growth curves of both CM-Dil-labelled and unlabelled ADSCs were sigmoidal in shape. The first 2 days were an incubation period, logarithmic growth began on the second day, and cell growth peaked on the sixth day, followed by a plateau period. The growth curves for CM-Dil-labelled and unlabelled ADSCs were similar (Fig. 4B). To evaluate the differentiation potential of ADSCs, we induced ADSCs to differentiate into adipogenic lineages that were identified by Oil Red 0 and into osteogenic lineages that were identified by Alizarin Red S (Fig. 4C-F). The results indicated that ADSCs have multidirectional differentiation potential.

\section{ADSCs transdifferentiated into epithelial stem cells}

Previous studies have shown that p63, $\alpha 6$-integrin and CD34 are markers of epithelial stem cells [12-15]. Merged fluorescence images of skin tissues demonstrated that the epithelial cell markers p63, $\alpha 6$-integrin and CD34 were coexpressed in ADSCs (Fig. 5). Thus, ADSCs could transdifferentiate into epithelial stem cells in photoaged skin. In addition, we found that the transferred ADSCs labelled with CM-Dil survived in the skin tissue for more than 6 weeks (Fig. 5).

\section{$B M P 4$, rather than BMP2, was a key factor in the photoaging process}

Western blotting results showed that Bmp4 expression was significantly lower in UVBtreated skin than in normal skin $(P<0.05)$, while Bmp4 expression was significantly higher in UVB+ADSCs-treated skin than in UVB-treated skin $(P<0.05)$ (Fig. 6ADI). The expression of BMP4 was downregulated by UVB irradiation, and ADSCs upregulated the low expression of BMP4 induced by UVB irradiation. Compared with Bmp4 expression, Bmp2 expression was not significantly changed among the three groups $(P>0.05)$, which was confirmed by both Western blot and RT-PCR analyses (Fig. 6ABCH). 


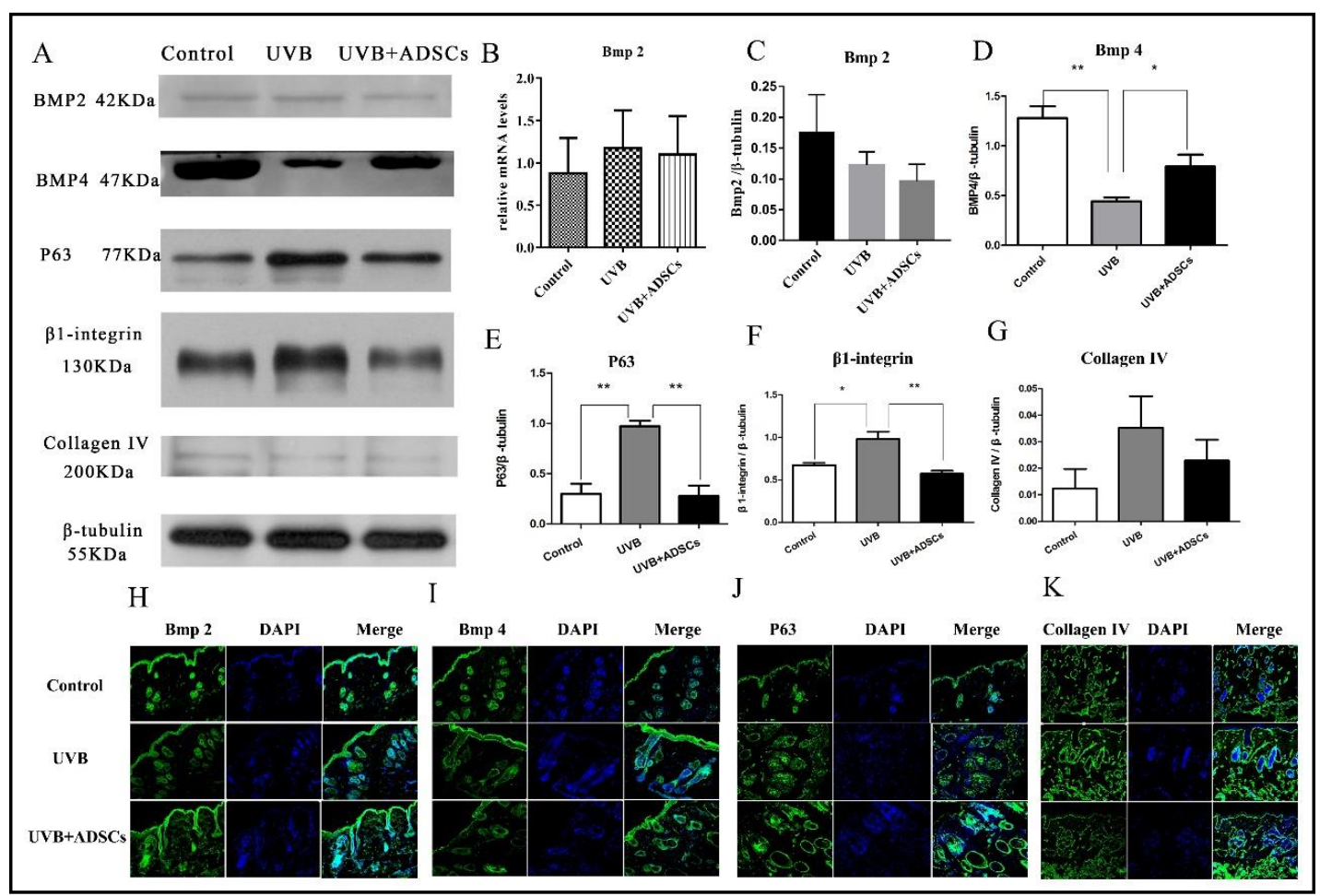

Fig. 6. (ADI) Expression of Bmp4 in the three groups. Western blot analysis showed that ADSCs transplantation upregulated the down-expression of Bmp4 induced by UVB irradiation. Merged fluorescence images demonstrate that Bmp4 is expressed in the three groups. (ABCH) Expression of Bmp2 in the three groups. The results of both Western blotting and RT-PCR show that Bmp2 expression is not significantly different among the three groups. Merged fluorescence images demonstrate that Bmp2 is expressed in the three groups. (AGK) Expression of Collagen IV in the three groups. Western blot analysis showed no significant changes among the three groups. Merged fluorescence images demonstrate that Collagen IV is expressed in the three groups. (AEFJ) Expression of p63 and $\beta 1$-integrin in the three groups. Western blot analysis showed that ADSCs transplantation downregulated the overexpression of p63 and $\beta 1$-integrin induced by UVB irradiation. $\left({ }^{*} \mathrm{P}<0.05, * * \mathrm{P}<0.01\right)$.

\section{Effect of ADSCs on the expression of downstream factors of Bmp4 signalling}

In this study, bioinformatic analysis showed that Smad1, MAPK1 and Nanog were all downstream factors of the Bmp4 pathway, and thus, they were analysed via RT-PCR. Smad1 and MAPK1 levels in the UVB group were significantly lower than those in the control group $(P<0.05)$, while Nanog expression was increased in the UVB group $(P<0.05)$ (Fig. 7DEF). ADSCs graft significantly improved the low expression of Smad1 and MAPK1, as well as the high expression of Nanog, in photoaged skin $(P<0.05)$ (Fig. 7DEF).

Effect of ADSCs on the expression of ageing-related factors in photoaged skin

ICAM-1, MMP13 and NFATC1 were analysed via RT-PCR. ICAM-1 and MMP13 expression in UVB-treated skin was significantly higher than that in the normal skin $(P<0.05)$, while ICAM-1 and MMP13 expression in UVB+ADSCs-treated skin was significantly lower than that in UVB-treated skin $(P<0.05)$ (Fig. 7A-B). The RT-PCR results for NFATC1 were opposite to those for MMP13 (Fig. 7C).

\section{ADSCs reduced UVB-induced $p 63$ and $\beta 1$-integrin overexpression}

In addition to its role as an epithelial marker, p63 is a basal transcription factor that promotes epidermal proliferation [16]. Expression of p63 was analysed via Western blotting. The results showed that p63 was significantly higher in UVB-treated skin than in normal skin and in UVB+ADSCs-treated skin. $(P<0.05)$ (Fig. 6AEJ). $\beta 1$-Integrin plays an essential KARGER 

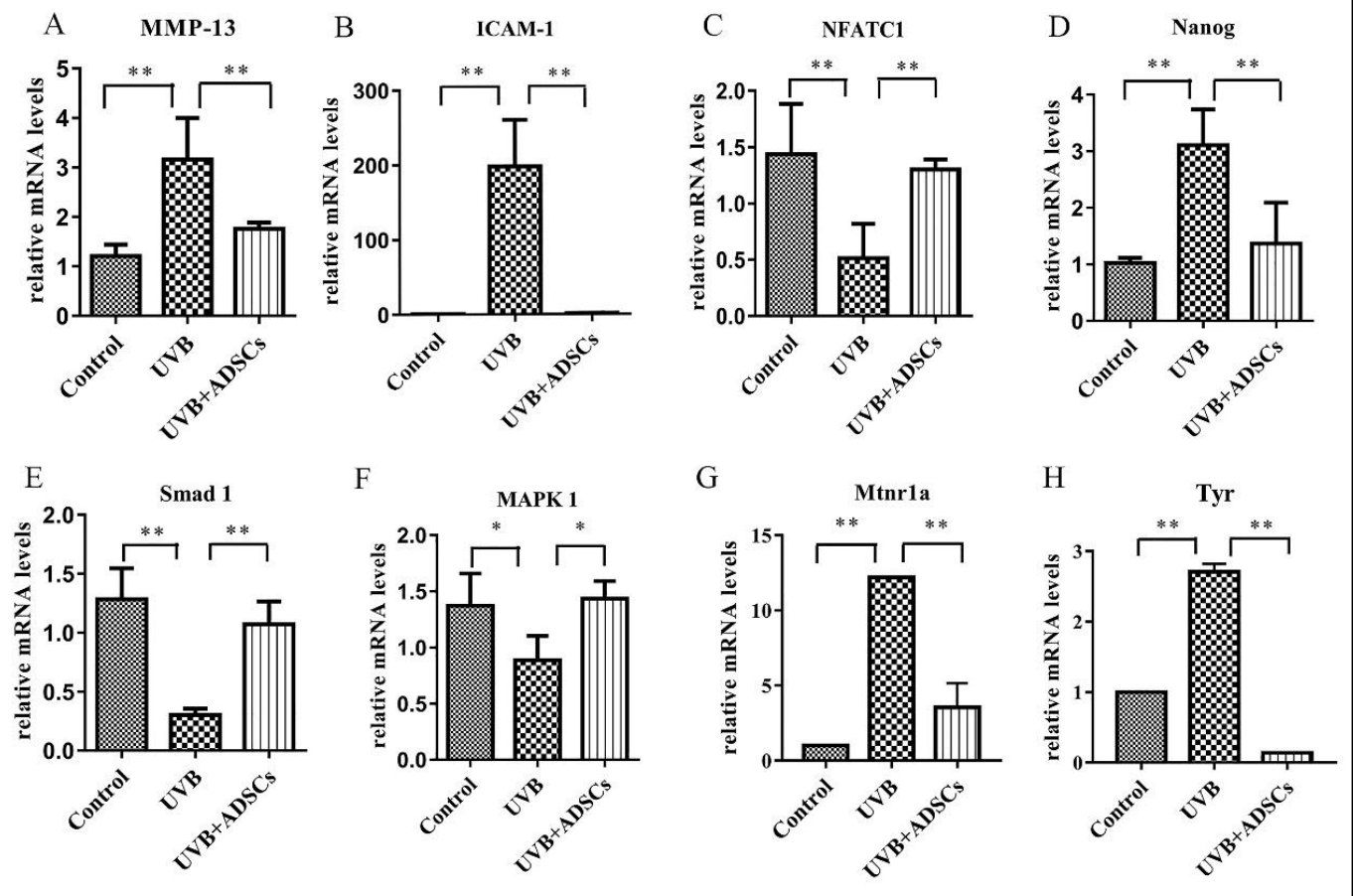

Fig. 7. (A) ADSCs transplantation downregulated the overexpression of MMP 13 induced by UVB irradiation. (B) ADSCs downregulated the overexpression of ICAM-1 caused by UVB irradiation. (C) ADSCs transplantation upregulated the downexpression of NFATC1 induced by UVB irradiation. (D) ADSCs transplantation downregulated the overexpression of Nanog induced by UVB irradiation. (E) ADSCs transplantation upregulated the downexpression of Smad1 induced by UVB irradiation. (F) ADSCs transplantation upregulated the downexpression of MAPK1 induced by UVB irradiation. (G-H) ADSCs transplantation downregulated the overexpression of Mtnr1a and Tyr induced by UVB irradiation. $\left({ }^{*} \mathrm{P}<0.05\right.$, $* * \mathrm{P}<0.01)$.

role in mediating interactions between the ECM and stem cells [4]. Western blotting showed that $\beta 1$-integrin was significantly higher in UVB-treated skin than in normal skin and in UVB+ADSCs-treated skin $(P<0.05)$ (Fig. 6AF). Thus, ADSCs reduced the p63 and $\beta 1$-integrin overexpression caused by UVB irradiation.

\section{Effect of ADSCs on Collagen IV expression in photoaged skin}

Immunofluorescence and Western blot analyses were used to assess Collagen IV expression in the photoaging process. No significant differences were observed between the UVB group and the control group $(P>0.05)$, and ADSCs transplantation did not significantly alter Collagen IV expression in ageing skin $(P>0.05)$ (Fig. 6AGK).

\section{Effect of ADSCs on melanogenesis and tyrosinase expression}

RT-PCR analysis showed that UVB irradiation upregulated the expression of Mtnr1a and Tyr, and ADSCs transplantation downregulated the overexpression of Mtnr1a and Tyr, in contrast with the results observed for BMP4 (Fig. 7G-H). 


\section{Cellular Physiology Cell Physiol Biochem 2018;51:2456-2471

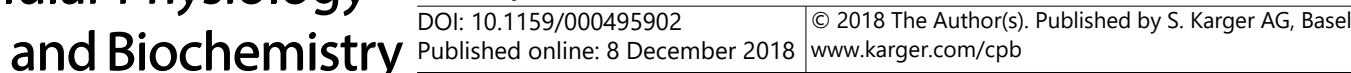 \\ Gong et al.: Remodelling of the Skin Stem Cell Niche During Photoaging}

\section{Discussion}

The effect of ageing on skin and hair follicle regeneration is likely controlled by stem cells and the local environment. Are the stem cells themselves or the local microenvironment responsible for skin cell behaviour? Giangreco A's study indicates that epidermal stem cells are present throughout life during normal ageing, suggesting that the local environment, rather than stem cells, affects skin ageing [17]. Whether the mechanism of photoaging is the same as that of natural ageing is unknown. In this study, the hair follicle stem cell niche was chosen to explore changes during photoaging and after transfer of ADSCs. The hair follicle is a great model with which to study stem cell biology because it is one of the few structures that can regenerate cyclically throughout life [3]. Stem cell niches are mainly composed of structural factors and biochemical factors. Structural factors include the structure and mechanical characteristics of the ECM. Biochemical factors include ECM adhesion factors and free cytokines.

In contrast with those of the SKH1 and BALB/c mice, the UVB-exposed female C57BL /6J mouse skin samples showed characteristics similar to those of photodamaged human skin [18]. Therefore, the experiments in this study were performed with female C57BL/6] mice.

An important barrier in cell therapy remains the low engraftment rate of transplanted cells, which diminishes the efficiency of cell therapy. Previous studies have suggested that transplanted ADSCs have very low retention in the later stages of transplantation. Remarkably, in our study, the injected CM-Dil-labelled ADSCs were detectable after six weeks. The viability of cells postinjection is critical to the success of injectable cell-based therapies [19]. However, transplanted cells cannot be entirely responsible for the beneficial effect on photoaging; a paracrine effect is more likely to explain the functional results. In hair follicle niches, BMP signalling acts as an important extrafollicular factor that regulates hair follicle regeneration in cooperation with intrafollicular Wnt/ $\beta$-catenin signalling [20]. Periodic BMP signalling inhibits the development and morphogenesis of hair follicles [21]. Both Bmp2 and Bmp4 are expressed in late anagen and refractory telogen and are lost in competent telogen [7]. However, the dynamic changes in Bmp2 and Bmp4 are different in ageing skin. Keyes's study indicates that Bmp2 levels increase in ageing mice and affect HFSC activation [22]. The upregulation of BMP in the natural ageing process slows hair follicle cycles and results in less hair [18]. In ageing mice, the regeneration cycle can be delayed by overexpression of intradermal inhibitors, such as BMP-2, DKK-1 and SFRP-4[3]. We examined the expression of BMP family members in mice treated with UVB irradiation. Interestingly, the expression of BMP4 decreased dramatically, while no significant change was found in BMP2 expression. These findings suggest that Bmp2 expression is regulated by programmed genes. We also found that Bmp4 showed the opposite expression trend in photoaging compared with that in natural ageing. This indicates a difference in the BMP signalling pathway between endogenous ageing and exogenous ageing. We suggest that UVB exposure acts as an external injury and causes an oxidative stress response in the skin. Downregulation of Bmp4 expression reduced the inhibitory effect on epithelial stem cells and HFSCs, which increased skin cycles and hair follicle formation. Mice in the UVB group showed epidermal thickening, a typical photoaging characteristic. Unexpectedly, there were local hair-loss zones in the UVB exposure areas in mice. We hypothesized that the disordered thickening of the epidermis hindered the hair from penetrating the skin, resulting in an absence of localized hair growth, which is also a photoaging characteristic. ADSCs transplantation upregulated Bmp4 expression, indicating that ADSCs could regulate stem cell behaviour by affecting the expression of macrofactors in niches. We suggest that downregulation of Bmp4 expression is a compensatory response of the skin to UVB damage. However, whether Bmp4 expression changes dynamically with prolonged UVB exposure requires further study.

BMPs mediate their biological effects via two pathways, BMP-Smad and BMP-Mapk [7]. BMPs regulate the transcription of BMP-responsive genes, including Smad1/Smad5/Smad8, via the Smad pathway [23]. The Mapk pathway plays an important role in hair cycle control, 
regulating the quiescence of HFSCs and catagen entry [24]. In our study, bioinformatics analysis showed that Smad1 and MAPK1 are specific downstream genes of Bmp4. RT-PCR results demonstrated that Smad1 and MAPK1 had the same expression trend as Bmp4 in the three groups. The Ras-MAPK pathway is stimulated in response to trauma to trigger a hyperproliferative response and cell migration. In the UVB group, MAPK1 downregulation may provide an explanation for the weakened wound healing ability of photoaged skin. In natural ageing, age-related hair damage is mostly associated with increased BMP/NFATc1 signalling in aged HFSCs [22]. Keyes et al. indicated that NFATc1 is elevated in aged HFSCs, resulting in ageing hair follicles with delayed entry into anagen during HFSCs activation [22]. Moreover, BMP-regulated Nfatc1 activity is a key contributor to the enhanced internal threshold that ageing HFSCs display once challenged with anagen cues [23]. We found that NFATc1 downregulation enhanced the hair cycles and increased hair formation in the UVB group. The ADSCs transplantation improved the low expression of Bmp4 and its related factors. These findings indicate that ADSCs can enhance the function of epithelial and hair follicle stem cells, as well as the damage repair ability of the skin in photoaging.

Bioinformatic analysis showed that Nanog was also a specific downstream factor of Bmp4. However, in contrast to the findings for Smad1 and MAPK1, RT-PCR results indicated that Nanog showed the opposite expression trend. As a core member of the transcriptional circuitry, Nanog helps to maintain the pluripotent state of stem cells [25]. In the UVB group, the high expression of Nanog increased the pluripotency and stemness of epithelial stem cells and HFSCs. Skin cells would then tend to overproliferate, which may provide an explanation for UVB-induced skin cancer. He et al. showed that the expression of Nanog in goat hair follicle stem cells (gHFSCs) was higher than that in goat adipose-derived stem cells (gADSCs), indicating that the stemness and pluripotency of gHFSCs was stronger than that of gADSCs [26]. We suggest that downregulation of Nanog after grafting of ADSCs may reduce the stemness and pluripotency of skin stem cells and promote differentiation in related cell repair injuries.

The ECM is essential for maintaining niche structure and function. One characteristic of ageing skin is changes in ECM composition [27]. MMPs can specifically degrade ECM proteins, resulting in wrinkles in the skin. Pittayapruek et al. showed that ECM degradation is the initial step in tumour cell invasion in photocarcinogenesis [28]. In addition, MMPs are involved in angiogenesis-mediated promotion of cancer cell migration and growth [28]. UVB photons are approximately 1000 times more energetic than UVA photons and are responsible for sunburn, pigmentation and photocarcinogenesis after sun exposure [18]. ADSCs downregulate the overexpression of ICAM-1 caused by UVB exposure and inhibit skin inflammation induced by UVB[9]. In the UVB group, MMP13 expression was significantly increased, which may explain why excessive UVB exposure can cause skin cancer. ADSCs transplantation reduced the increased MMP13 expression, indicating that ADSCs may prevent and ameliorate skin cancer by improving MMP13 expression. Increased MMP levels initiate Collagen I and III degradation [29]. As an important component of the ECM, Collagen IV regulates cell behaviours by changing the stiffness of stem cell niches [27]. Choi et al. demonstrated that Collagen IV plays an important role in shaping the niche of epidermal stem cells [14]. However, in our study, there was no significant change in the expression of Collagen IV in the three groups. We suggest that Collagen IV does not participate in niche alteration and remodelling during photoaging.

UVB irradiation is well known to stimulate melanogenesis and tyrosinase via BMP4 and its receptor BMP-1b in the skin. BMP4 markedly inhibits melanogenesis by downregulating tyrosinase expression and activity to below baseline levels [30]. The mechanism of this BMP4 effect on tyrosinase and ultimately on melanogenesis involves modest decreases in the tyrosinase transcription rate and mRNA stability. UVB irradiation stimulates melanogenesis by downregulating the mRNA of BMP receptor-1B in melanocytes [31]. In our study, we found that UVB irradiation upregulated the expression of melatonin receptor $1 \mathrm{~A}$ and tyrosinase and ADSCs transplantation reduced the expression of melatonin receptor $1 \mathrm{~A}$ and tyrosinase, in contrast with the results observed for BMP4. These data suggest that BMP4 activators

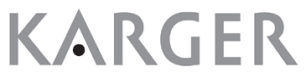




\section{Cellular Physiology Cell Physiol Biochem 2018;51:2456-2471 and Biochemistry \begin{tabular}{c|c} 
DOl: 10.1159/000495902 & Published 2018 The Author(s). Published by S. Karger AG, Basel \\
\cline { 2 - 4 } & www.karger.com/cpb
\end{tabular} \\ Gong et al.: Remodelling of the Skin Stem Cell Niche During Photoaging}

may play novel roles as melanogenesis and melanin-transfer inhibitors.

This study showed that UVB reduced the function of stem cell niches by degrading ECM proteins and repressing the expression of Bmp4 and its related factors, leading to various signs of photoaging in the skin. ADSCs transplantation reversed these changes and remodelled the niches of skin stem cells to improve skin photoaging. However, if the effect of ADSCs is simply understood as a paracrine mechanism, it is less likely to represent a sustainable mechanism responsible for the lasting contribution of ADSCs to skin rejuvenation. Hwang I et al. found that the introduction of either DPCs or HFSCs to the dermal layers of mouse skin triggers the formation and growth of hair follicles [32]. Marinkovic $\mathrm{M}$ et al. proposed that the tissue-specific effects of ECMs on MSCs proliferation and differentiation may reflect the ability of the bone marrow ECM and adipose ECM to recapitulate the physical characteristics of the MSC niches found in bone marrow and adipose tissue [33]. In our study, ADSCs were injected into the skin, and the grafted ADSCs under the influence of the native extracellular matrix in the skin expressed epithelial stem cell markers, such as p63, $\alpha 6$-integrin and CD34, which suggested that ADSCs can transdifferentiate into skin stem cells. Moreover, the grafted ADSCs survived in the skin tissue for more than 6 weeks. Prolonged survival increases the ability of ADSCs to ameliorate skin photoaging. ADSCs primarily function through paracrine mechanisms and transdifferentiation. Therefore, we believe that ADSCs do not remodel stem cell niches through a single mechanism. ADSCs may play a role through the paracrine pathway in the early stage and likely play a role through transdifferentiation into epithelial stem cells in the late stage of transplantation. However, determining how ADSCs transdifferentiate into skin stem cells requires further study.

\section{Conclusion}

The increasing demand for skin rejuvenation has contributed to a continuous development in the understanding of skin ageing mechanisms. Our study indicates that UVB exposure reduces the capacities of skin stem cells by altering the microenvironmental structure and macroenvironmental signals in skin stem cell niches. ADSCs reversed the typical photoaging signs by remodelling skin stem cell niches through BMP4 pathway modulation and transdifferentiation into skin stem cells. In addition, the ADSCs survived for more than 6 weeks in skin tissue after ADSCs transfer. These discoveries provide theoretical support for the treatment of skin photoaging with ADSCs transplantation.

\section{Acknowledgements}

This work was supported by the National Science Foundation of China (grant no. 81771994) and the Innovation Science Foundation of Harbin Medical University (no. 2017LCZX49).

\section{Disclosure Statement}

The authors declare that no conflicts of interest exists. 


\section{Cellular Physiology Cell Physiol Biochem 2018;51:2456-2471 \begin{tabular}{ll|l} 
and BiOChemistry & $\begin{array}{l}\text { DOI: 10.1159/000495902 } \\
\text { Published online: 8 December } 2018\end{array}$ & $\begin{array}{l}\text { ( } 2018 \text { The Author(s). Published by S. Karger AG, Basel } \\
\text { www.karger.com/cpb }\end{array}$ \\
\hline
\end{tabular}}

\section{References}

1 Lopez-Otin C, Blasco MA, Partridge L, Serrano M, Kroemer G: The hallmarks of aging. Cell 2013;153:11941217.

- Fuchs E: Scratching the surface of skin development. Nature 2007;445:834-842.

3 Chen CC, Plikus MV, Tang PC, Widelitz RB, Chuong CM: The Modulatable Stem Cell Niche: Tissue Interactions during Hair and Feather Follicle Regeneration. J Mol Biol 2016;428:1423-1440.

4 Gattazzo F, Urciuolo A, Bonaldo P: Extracellular matrix: a dynamic microenvironment for stem cell niche. Biochim Biophys Acta 2014;1840:2506-2519.

5 Mammoto T, Ingber DE: Mechanical control of tissue and organ development. Development 2010;137:1407-1420.

-6 Plikus MV, Baker RE, Chen CC, Fare C, de la Cruz D, Andl T, Maini PK, Millar SE, Widelitz R, Chuong CM: Selforganizing and stochastic behaviors during the regeneration of hair stem cells. Science 2011;332:586-589.

7 Plikus MV, Mayer JA, de la Cruz D, Baker RE, Maini PK, Maxson R, Chuong CM: Cyclic dermal BMP signalling regulates stem cell activation during hair regeneration. Nature 2008;451:340-344.

8 Ganceviciene R, Liakou AI, Theodoridis A, Makrantonaki E, Zouboulis CC: Skin anti-aging strategies. Dermatoendocrinol 2012;4:308-319.

- 9 Li H, Fu X: Mechanisms of action of mesenchymal stem cells in cutaneous wound repair and regeneration. Cell Tissue Res 2012;348:371-377.

10 Zhang JA, Yin Z, Ma LW, Yin ZQ, Hu YY, Xu Y, Wu D, Permatasari F, Luo D, Zhou BR: The protective effect of baicalin against UVB irradiation induced photoaging: an in vitro and in vivo study. PLoS One 2014;9:e99703.

11 Cho JM, Lee YH, Baek RM, Lee SW: Effect of platelet-rich plasma on ultraviolet b-induced skin wrinkles in nude mice. J Plast Reconstr Aesthet Surg 2011;64:e31-39.

12 Shin JW, Choi HR, Nam KM, Lee HS, Kim SA, Joe HJ, Kazumi T, Park KC: The Co-Expression Pattern of p63 and HDAC1: A Potential Way to Disclose Stem Cells in Interfollicular Epidermis. Int J Mol Sci 2017;18:E1360.

13 Derby BM, Dai H, Reichensperger J, Cox L, Harrison C, Cosenza N, Yang M, Bueno RA, Neumeister MW: Adipose-derived stem cell to epithelial stem cell transdifferentiation: a mechanism to potentially improve understanding of fat grafting's impact on skin rejuvenation. Aesthet Surg J 2014;34:142-153.

14 Choi HR, Byun SY, Kwon SH, Park KC: Niche interactions in epidermal stem cells. World J Stem Cells 2015;7:495-501.

15 Hu HM, Zhang SB, Lei XH, Deng ZL, Guo WX, Qiu ZF, Liu S, Wang XY, Zhang H, Duan EK: Estrogen leads to reversible hair cycle retardation through inducing premature catagen and maintaining telogen. PLoS One 2012;7:e40124.

16 Yang A, Schweitzer R, Sun D, Kaghad M, Walker N, Bronson RT, Tabin C, Sharpe A, Caput D, Crum C, McKeon F: p63 is essential for regenerative proliferation in limb, craniofacial and epithelial development. Nature 1999;398:714-718.

17 Giangreco A, Qin M, Pintar JE, Watt FM: Epidermal stem cells are retained in vivo throughout skin aging. Aging Cell 2008; 7:250-259.

18 Sharma MR, Werth B, Werth VP: Animal models of acute photodamage: comparisons of anatomic, cellular and molecular responses in C57BL/6J, SKH1 and Balb/c mice. Photochem Photobiol 2011;87:690-698.

19 Zhang S, Dong Z, Peng Z, Lu F: Anti-aging effect of adipose-derived stem cells in a mouse model of skin aging induced by D-galactose. PLoS One 2014;9:e97573.

20 Chen CC, Murray PJ, Jiang TX, Plikus MV, Chang YT, Lee OK, Widelitz RB, Chuong CM: Regenerative hair waves in aging mice and extra-follicular modulators follistatin, dkk1, and sfrp4. J Invest Dermatol 2014;134:2086-2096.

21 Wang X, Tredget EE, Wu Y: Dynamic signals for hair follicle development and regeneration. Stem Cells Dev 2012;21:7-18.

22 Keyes BE, Segal JP, Heller E, Lien WH, Chang CY, Guo X, Oristian DS, Zheng D, Fuchs E: Nfatc1 orchestrates aging in hair follicle stem cells. Proc Natl Acad Sci U S A 2013;110:E4950-4959.

23 Botchkarev VA, Sharov AA: BMP signaling in the control of skin development and hair follicle growth. Differentiation 2004;72:512-526. 


\section{Cellular Physiology Cell Physiol Biochem 2018;51:2456-2471 \begin{tabular}{l|l|l} 
DOl: 10.1159/000495902 & $\begin{array}{l}\text { O 2018 The Author(s). Published by S. Karger AG, Basel } \\
\text { www.karger.com/cpb }\end{array}$ \\
\hline
\end{tabular}}

24 Akilli Ozturk O, Pakula H, Chmielowiec J, Qi J, Stein S, Lan L, Sasaki Y, Rajewsky K, Birchmeier W: Gab1 and Mapk Signaling Are Essential in the Hair Cycle and Hair Follicle Stem Cell Quiescence. Cell Rep 2015;13:561-572.

25 Chambers I, Silva J, Colby D, Nichols J, Nijmeijer B, Robertson M, Vrana J, Jones K, Grotewold L, Smith A: Nanog safeguards pluripotency and mediates germline development. Nature 2007;450:1230-1234.

-26 He N, Dong Z, Zhu B, Nuo M, Bou S, Liu D: Expression of pluripotency markers in Arbas Cashmere goat hair follicle stem cells. In vitro Cell Dev Biol Anim 2016;52:782-788.

-27 Rock K, Joosse SA, Muller J, Heinisch N, Fuchs N, Meusch M, Zipper P, Reifenberger J, Pantel K, Fischer JW: Chronic UVB-irradiation actuates perpetuated dermal matrix remodeling in female mice: Protective role of estrogen. Sci Rep 2016;6:30482.

28 Pittayapruek P, Meephansan J, Prapapan 0, Komine M, Ohtsuki M: Role of Matrix Metalloproteinases in Photoaging and Photocarcinogenesis. Int J Mol Sci 2016;17:E868.

29 Fisher GJ, Wang ZQ, Datta SC, Varani J, Kang S, Voorhees JJ: Pathophysiology of premature skin aging induced by ultraviolet light. N Engl J Med 1997;337:1419-1428.

-30 Singh SK, Abbas WA, Tobin DJ: Bone morphogenetic proteins differentially regulate pigmentation in human skin cells. J Cell Sci 2012;125:4306-4319.

-31 Yaar M, Wu C, Park HY, Panova I, Schutz G, Gilchrest BA: Bone morphogenetic protein-4, a novel modulator of melanogenesis. J Biol Chem 2006;281:25307-25314.

-32 Hwang I, Choi KA, Park HS, Jeong H, Kim JO, Seol KC, Kwon HJ, Park IH, Hong S: Neural Stem Cells Restore Hair Growth Through Activation of the Hair Follicle Niche. Cell Transplant 2016;25:1439-1451.

-33 Marinkovic M, Block TJ, Rakian R, Li Q Wang E, Reilly MA, Dean DD, Chen XD: One size does not fit all: developing a cell-specific niche for in vitro study of cell behavior. Matrix Biol 2016;52-54:426-441. 